\title{
Holographic Pyramid Using Integral Photography Suitable for Displaying Tall Objects
}

\author{
Shohei Anraku' ${ }^{1}$, Toshiaki Yamanouchi ${ }^{1}$, Nahomi Maki², Kazuhisa Yanaka1 \\ ${ }^{1}$ Kanagawa Institute of Technology \\ 1030 Shimo-ogino, Atsugi-shi, Kanagawa-ken, 243-0292 Japan \\ yama@ic.kanagawa-it.ac.jp; s1785004@cce.kanagawa-it.ac.jp, yanaka@ic.kanagawa-it.ac.jp \\ ${ }^{2}$ Tokyo University of the Arts (Bankokubashi Campus) \\ 4-32 Kaigan-dori, Naka-ku, Yokohama-shi, Kanagawa-ken, 231-0002 Japan \\ maki.nahomi@fm.geidai.ac.jp
}

\begin{abstract}
This study proposes a new holographic pyramid in which a 3D object, such as a human, can be displayed approximately 1.4 times larger even if the size of the liquid crystal display used is the same. The 3D object appears as if it is floating in the pyramid, which is typically made of plastic, and the object itself has a parallax in both horizontal and vertical directions because integral photography is used in the system.
\end{abstract}

Keywords: 3D Display, Autostereoscopic, Integral Photography, Holographic Pyramid, Game Engine, Unity.

\section{Introduction}

Holographic pyramid is a unique 3D display consisting of a liquid crystal display (LCD) and a quadrangular pyramid made of transparent material. The display can be observed from all $360^{\circ}$, and $3 \mathrm{D}$ objects such as aquatic creatures appear to float in the center of the pyramid. In a conventional holographic pyramid, the displayed object is planar. However, when an integral photography (IP)-type display [1] is used instead of a normal 2D display, parallax occurs within the 3D object itself and a higher reality is obtained [2]. IP is an excellent autostereoscopic display method because it has both horizontal and vertical parallax.

In conventional holographic pyramid systems, one side of a quadrangular pyramid is placed so that it is parallel to one side of the LCD (Fig. 1). However, this method is not suitable for displaying a tall object, such as a character, because the height of the displayed character cannot exceed half of the short side of the LCD (Fig. 3).

If the square pyramid is rotated $45^{\circ}$ with respect to the LCD (Fig. 2), then the size of the displayed character can be increased (Fig. 4).

In holographic pyramids that do not use IP, the image displayed on the LCD is an ordinary 2D image, which can easily be rotated to view the image shown in Fig. 4 by using digital image processing techniques. However, in the holographic pyramid using IP, a directionality exists in the IP image shown in Fig. 5. Therefore, the rotated IP image cannot be viewed stereoscopically because the direction of the IP image and the direction of the fly's eye lens are no longer coincident. Therefore, the present study proposes a method that is also applicable to holographic pyramid using IP.

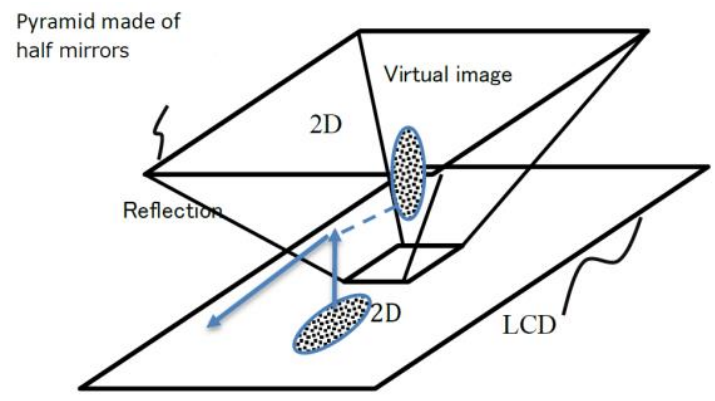

Fig. 1: Conventional holographic pyramid system. 


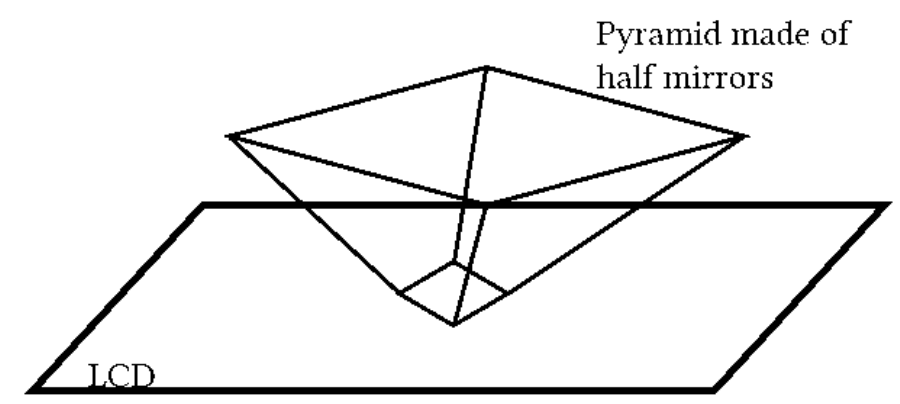

Fig. 2: Holographic pyramid with pyramid rotated $45^{\circ}$.
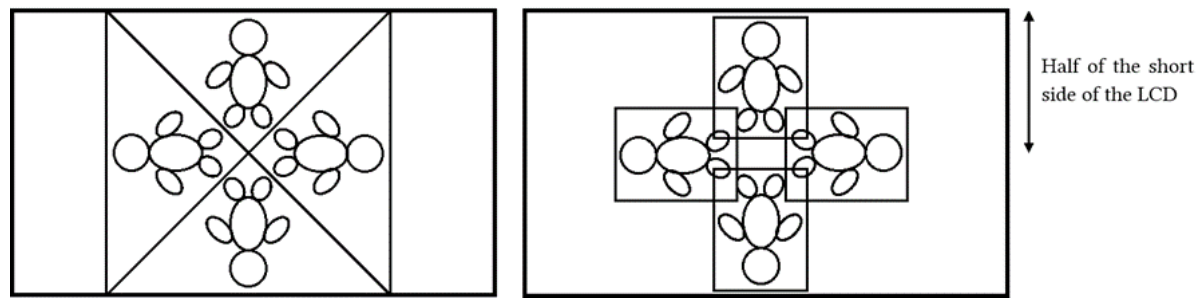

Fig. 3: Example of display methods of a conventional holographic pyramid.

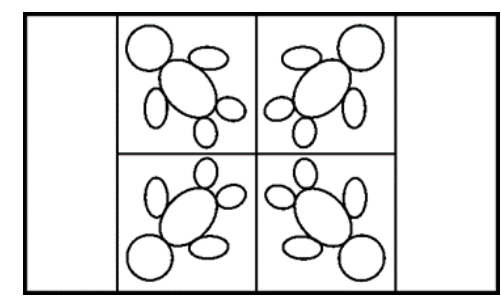

Fig. 4: Display method of the proposed holographic pyramid.

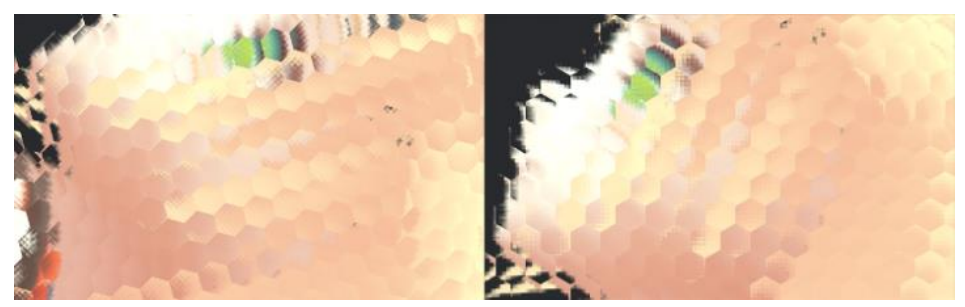

Fig. 5: Directionality in IP images. (The image consists of a large number of minute hexagons, each corresponding to a hexagonal convex lens of a fly's eye lens.) OUTJ/UCL.

\section{Method}

Although our method itself does not rely on a specific development environment, the case is described using Unity 5 because Unity is suitable for the application development of IP [3].

Fig. 6 shows a general IP image synthesis method for a holographic pyramid using Unity [4]. In the lower right of the figure, four camera arrays surround the subject. Each camera array consists of $8 \times 8=64$ virtual cameras placed at the front, back, left, and right of a subject that is to be displayed in the pyramid. A memory area, called a render texture, of $4096 \times 4096$ pixels is allocated to each camera array. Each render texture is divided into $8 \times 8=64$ viewports $(512 \times$ 512 pixels each), and the images seen from the different cameras are drawn.

As shown in the middle left of Fig. 6, four planes (front, left, right, and back) are present, and each render texture is texture-mapped to the corresponding plane.

Let us assume that the field of view of each camera is a square. To display the subject as large as possible, it is sufficient to rotate the character of the subject by $45^{\circ}$. IP has a parallax in all directions, so even if the subject is rotated, 
it can be displayed stereoscopically. However, if the character is rotated, then the direction will change when it is seen from other directions. Therefore, each camera array is rotated without rotating the subject (Fig. 7).

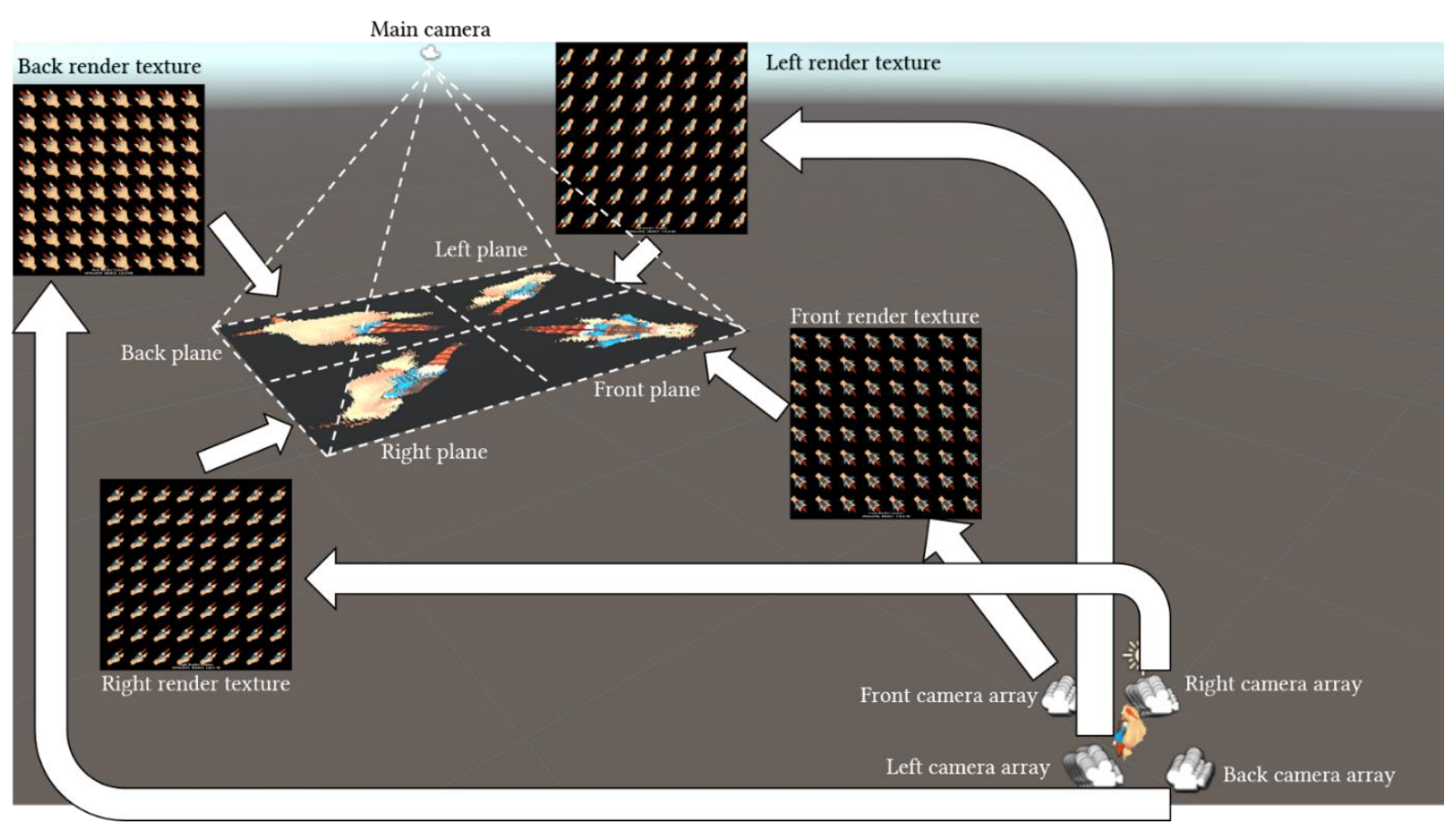

Fig. 6: IP image synthesis method using Unity. OUTJ/UCL.
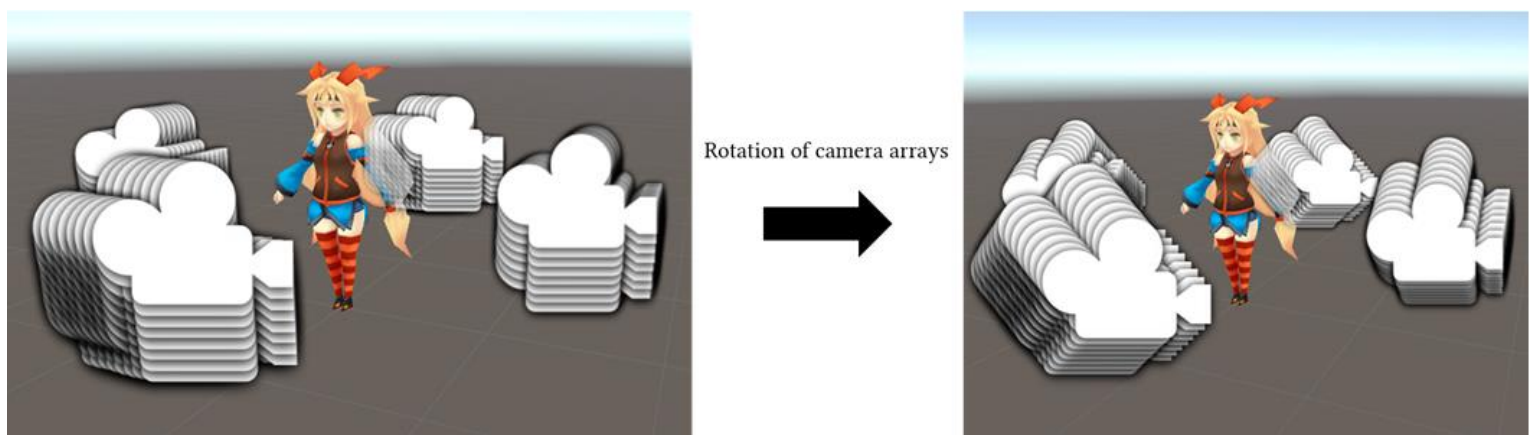

Fig. 7: Rotation of each camera array. OUTJ/UCL.

\section{Experiment}

\subsection{Experimental Equipment}

For the experiment, we used a 13.5-inch PC with a $3000 \times 2000$ resolution Microsoft Surface Book. The fly's eye lens were developed by Fresnel Technology, and the pyramid was made of half mirrors. This application was developed with Unity. A 3D model of Low-Poly Unity-Chan [5] was used to increase the rendering speed.

\subsection{Experimental Result}

When a conventional holographic pyramid is used to display a tall object like a character, the IP image displayed on the LCD is as shown in Fig. 8 (a), and the displayed character size is as small as shown in Fig. 9 (a). By using our technology where the pyramid is rotated $45^{\circ}$ against the LCD, the IP image displayed on the LCD is as shown in Fig. 8 (b), and the displayed character size increases to approximately 1.4 times the previous size, even when the same LCD size is used, as shown in Fig. 9 (b). 


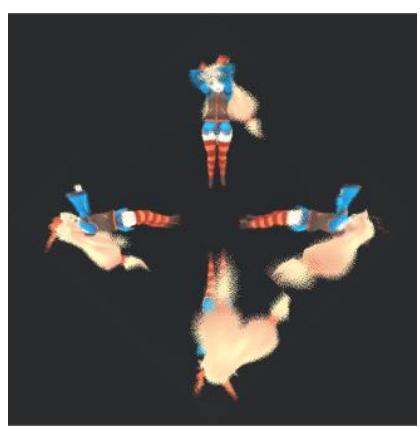

(a) Conventional method

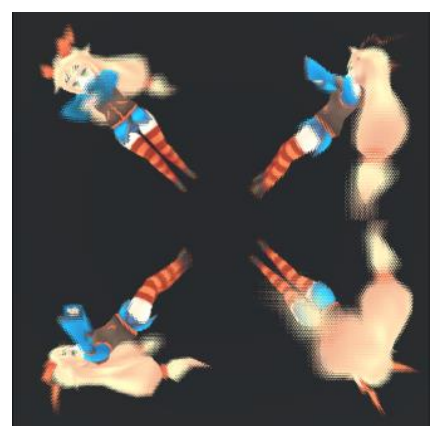

(b) Proposed method

Fig. 8: Comparison of IP images displayed on the LCD. OUTJ/UCL.

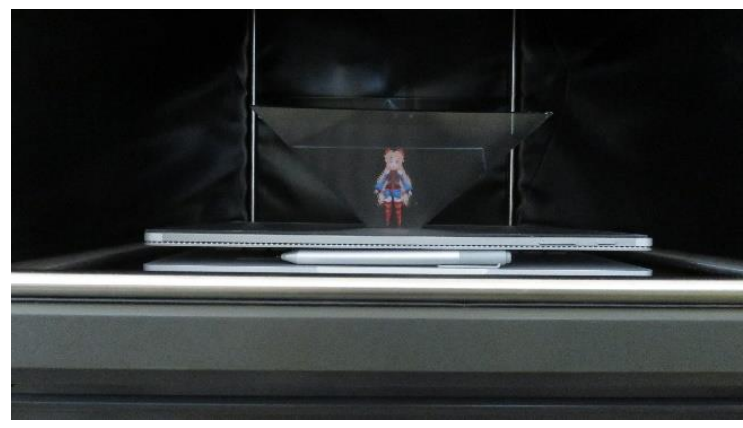

(a) Conventional method

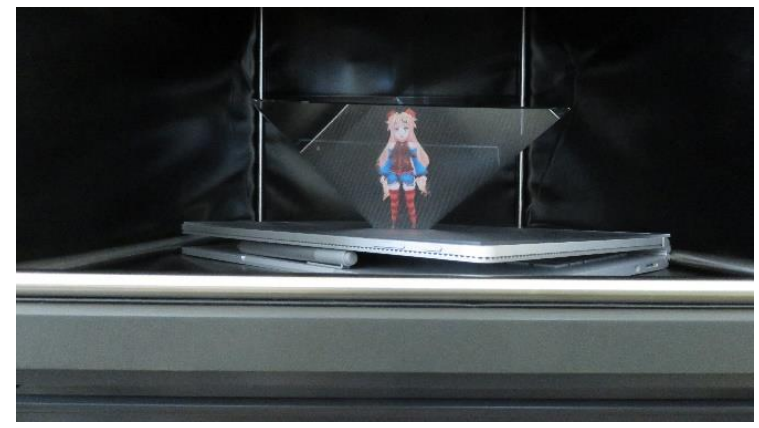

(b) Proposed method

Fig. 9: Comparison of character sizes displayed on the holographic pyramid. @UTJ/UCL.

\section{Conclusions}

Holographic pyramid using IP is a wonderful 3D display in which a 3D object with both horizontal and vertical parallax looks like it is floating in the pyramid. The display can be observed from all four directions without the need to wear stereo glasses. This study proposes a method to display a tall object, such as a person, as large as possible within the range of the square display area. Although the size of the LCD remains the same, tall objects are displayed approximately 1.4 times larger. A delightful side effect of the proposed method is that the 3D image quality improves because the number of pixels used to display the object increases.

\section{Acknowledgement}

This work was supported by KAKENHI Grant Number 16K00284.

\section{References}

[1] M. G. Lippmann, "Epreuves, reversibles donnant la sensation du relief," in J. Phys., vol.4, pp. 821-825, 1908.

[2] T. Yamanouchi, N. Maki and K. Yanaka, "Holographic Pyramid Using Integral Photography," in Proceedings of EECSS'16, Paper No. MHCI 109, pp. 1-4. 2016.

[3] S. Anraku, F. Ishiwata, N. Maki, T. Yamanouchi and K. Yanaka, "Real-Time Integral Photography Using a Game Engine," in SIGGRAPH 2017 Posters, 2017.

[4] S. Anraku, T. Yamanouchi and K. Yanaka, "Real-Time Integral Photography Holographic Pyramid Using a Game Engine," in VISIGRAPP 2018 Proceedings, vol. 4, VISAPP, pp.603-607, 2018.

[5] Unity Technologies Japan, 2016. LowPolyUnityChan. [Online]. Available: http://unitychan.com/download/releaseNote.php?id=LowPolyUnityChan\&lang=en 